\title{
Highly Sensitive Rapid DNA Sensor Based on Light-induced Acceleration of Molecular Recognition
}

\author{
$\underline{\text { Takuya lida }}^{1,2^{*}}$, Yushi Nishimura ${ }^{1,2,{ }^{*}, \text { Mamoru Tamura }}{ }^{1,2}$, Keisuke Nishida $^{2,3}$, Syoji Ito ${ }^{4}$, \\ ${ }^{1}$ Department of Physical Science, Graduate School of Science, Osaka Prefecture University, Sakai, \\ Osaka 599-8570, Japan \\ ${ }^{2}$ Research Institute for Light-induced Acceleration System (RILACS), Sakai, Osaka 599-8570, Japan \\ ${ }^{3}$ Department of Applied Chemistry, Graduate School of Engineering, Osaka Prefecture University, \\ Sakai, Osaka 599-8570, Japan \\ *t-iida@p.s.osakafu-u.ac.jp
}

\begin{abstract}
:
Molecular recognition is a fundamental mechanism for the specific detection in various types of biosensor technology. Here, we theoretically and experimentally demonstrated the "Light-induced acceleration of molecular recognition", and succeeded in the rapid detection of zeptomole-level DNA. The formation of a submillimetre network-like structure was observed at desired position under within several minutes via the laser-induced hybridization of DNA-modified NPs and target DNA exhibiting a broadband optical spectrum. This finding describes a strategy for the remote control of hybridization of extremely small amounts of DNA (1/1000,000 of that without laser irradiation) as a trigger, and for not only rapid bottom-up production of macroscopic assembly with NPs even without PCR amplification, but also rapid and highly sensitive genetic screening without fluorescent labeling.
\end{abstract}

Key words: DNA, optical tweezers, biosensor, molecular recognition, photothermal convection

\section{Introduction}

The mechanism of molecular recognitions in various biological samples, for example, DNA double strand formation, antigen-antibody reaction, molecular imprinting are widely utilized for the specific detection [1]. Specific binding of probe biomolecules modified on nanoparticles (NPs) and target molecules leads to optically visible large assembly, which is promising for the detection of targets. If the NPs would be remotely assembled, the application range will be greatly extended. Previously, the lightinduced assembly of metallic NPs and the prominent spectral modulation due to the plasmonic superradiance was theoretically predicted $[2,3]$. Also, the enhancement of the light-induced force (LIF) and light-induced convection (LIC) due to such a collective effect of electrons in high-density metallic NPs was utilized for the macroscopic assembly [4]. In this contribution, paying attention to this mechanism, we try to theoretically and experimentally clarify the light-induced dynamics of probe NPs modified with single strand DNA and target DNA for highly efficient biosensing [5]. (a)

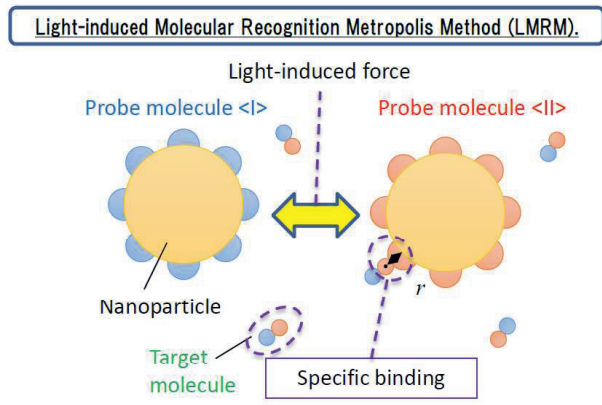

(b)

(c)
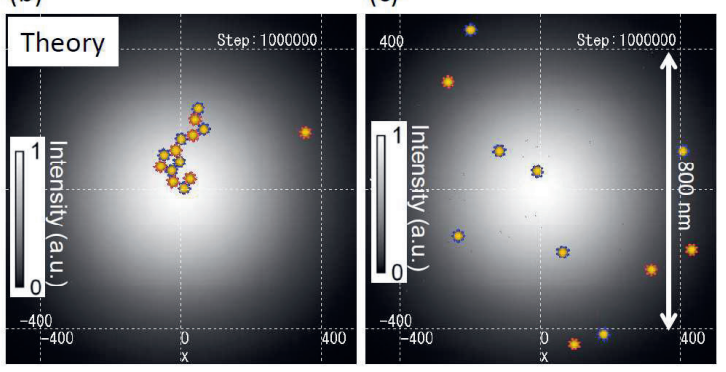

Figure 1. Simulation results of light-induced hybridization of DNA mediated by NPS with the model in Fig. 1 (After 50,000 Monte Carlo steps). Laser power was assumed to be $50 \mathrm{~mW}$. 


\section{Methods}

Our previous theoretical method for lightinduced assembly [3] was extended taking into account molecular recognition, "Light-induced molecular recognition Metropolis Method (LMRM)". With this method, the light-induced assembly process of probe NPs and target DNA molecules was evaluated in the model of Fig.1(a), where these NPs were modelled by spherical cells modified with DNA hemispheres. The corresponding experiment was performed for light-induced hybridization of DNA via the probe NPs under the irradiation of infrared (IR) laser near the air-liquid interface with the experimental setup in Fig.2(a).
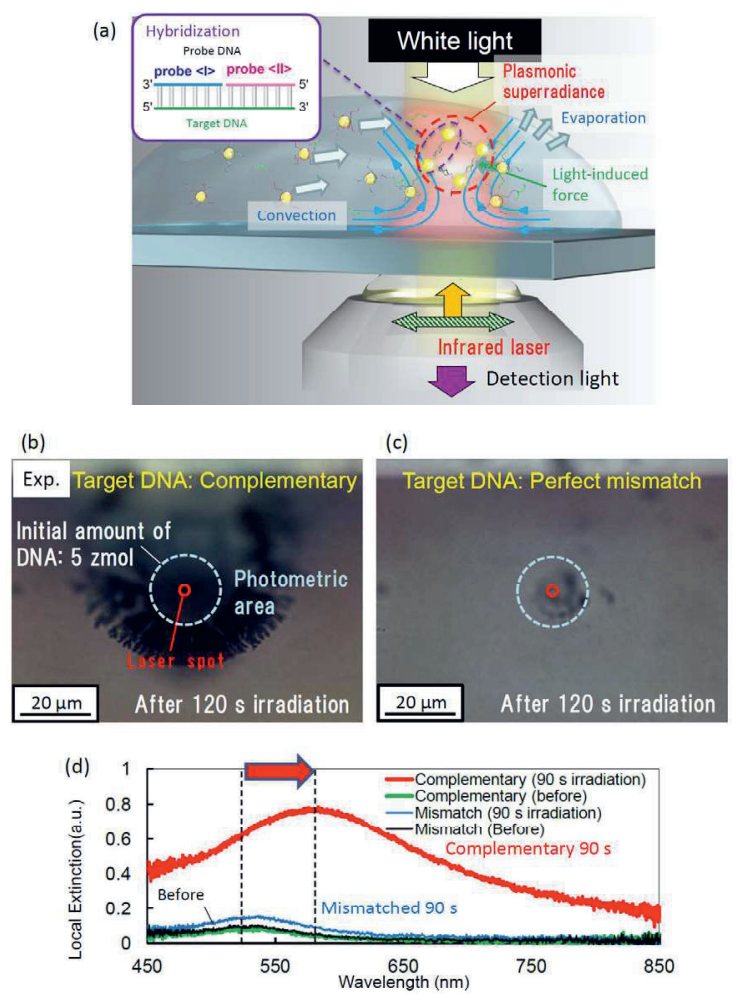

Figure 2. (a) Schematic image of experimental setup. (b) Optically assembled network structure of complementary target DNA and probe NPs. (c) Experimental result with Mismatched target DNA under the similar condition in (b). In both cases, laser power was $40 \mathrm{~mW}$, DNA concentration was $100 \mathrm{pM}$, and broken white circles are the photometric area. (d) Local extinction spectra for complementary and mismatched DNA before and after laser irradiation.

\section{Results and Discussion}

In LMRM calculation, many Au NPs of $30 \mathrm{~nm}$ in diameter were focused IR laser with spot diameter $1 \mu \mathrm{m}$, was performed (Fig.1). LIF accelerates the hybridization of the target complementary DNA and the probe DNA on the surface of probe NPs, and the assembled NPs via DNA were strongly trapped by gradient force around the high laser intensity region near the laser spot (Fig.1 (b)). On the other hand, in the case of mismatched target DNA, LIF on isolated Au NPs is too small and the Brownian motion induces the diffusion (Fig.1 (c)).

Next, constructing an experimental setup (Fig. 2(a)) with the similar laser condition such as wavelength, power, and spot diameter, it has been revealed that submillimetre network structure was optically assembled via the hybridization of small amount of DNA on the order of $\mathrm{zmol}$ in the photometric area within 2 minutes (Fig.2 (b)). As the comparative experiment, no assembled structure was observed in microtube even after 3 hours wituout laser irradiation for the same DNA concentration. Moreover, no macroscopic structure was produced with mismatched target DNA even under laser irradiation (Fig.3 (c)). From the distribution of network structure, not only LIF theoretically estimated in Fig. 1, but also the enhancement of photothermal effect also play a crucial role. Due to the high photothermal effect from large red-shift and broadening of localized surface plasmon resonance (Fig. 2(d)), strong LIC would accelerate the DNA double strand formation.

In conclusion, macroscopic network structure was produced by assembly of probe NPs via zmol-level DNA within only a few minutes. The clarified principle can be used for novel biosensor for not only DNA but also various types of nano-biomaterials via light-induced acceleration of molecular recognition processes.

\section{References}

[1] S. Tokonami, T. lida, et al., "Detection of Biomaterials and Bacteria Using Functionalized Nano-and Micro-Spaces," Review article in BUNSEKI KAGAKU, 64(10), 727-736 (2015).

[2] T. lida, "Control of Plasmonic Superradiance in Metallic Nanoparticle Assembly by Light-Induced Force and Fluctuations," J. Phys. Chem. Lett. 3(3), 332-336 (2012).

[3] S. Ito, et al., "Selective Optical Assembly of Highly Uniform Nanoparticles by DoughnutShaped Beams," Sci. Rep. 3, 3047(1-7) (2013).

[4] Y. Nishimura, S. Tokonami, T. lida, et al., "Control of Submillimeter Phase Transition by Collective Photothermal Effect," J. Phys. Chem. C 118(32), 18799-18804 (2014).

[5] T. lida, Y. Nishimura, M. Tamura, K. Nishida,S. Ito, S. Tokonami, "Submillimetre Network Formation by Light-induced Hybridization of Zeptomole-level DNA," Sci. Rep. 6, 37768(1-9) (2016). 